\title{
The phase portrait of the Hamiltonian system associated to a Pinchuk map
}

\author{
JOAN CARLES ARTÉS ${ }^{1}$, FRANCISCO BRAUN ${ }^{2}$ and JAUME LLIBRE ${ }^{3}$ \\ ${ }^{1,3}$ Departament de Matemàtiques, Universitat Autònoma de Barcelona, Edifici Cc, Campus de la UAB, \\ Bellaterra, Cerdanyola del Vallès, 08193 Barcelona, Catalonia, Spain \\ ${ }^{2}$ Departamento de Matemática, Universidade Federal de São Carlos, Rodovia Washington Luís, \\ Km 235, Caixa Postal 676, 13565-905 São Carlos, SP, Brazil
}

Manuscript received on October 22, 2017; accepted for publication on December 13, 2017

\begin{abstract}
In this paper we describe the global phase portrait of the Hamiltonian system associated to a Pinchuk map in the Poincaré disc. In particular, we prove that this phase portrait has 15 separatrices, five of them singular points, and 7 canonical regions, six of them of type strip and one annular.
\end{abstract}

Key words: center, global injectivity, real Jacobian conjecture, Pinchuk map.

\section{INTRODUCTION}

As far as we know, the simplest class of non-injective polynomial local diffeomorphisms of $\mathbb{R}^{2}$ are the Pinchuk maps, constructed by Pinchuk (1994). The existence of these maps disproves the real Jacobian conjecture, that a polynomial local diffeomorphism of $\mathbb{R}^{2}$ is globally injective. One open problem is to know what exactly fails in this conjecture.

One of the most known conditions for a local diffeomorphism to be a global one is that it is proper. The asymptotic variety of a map of $\mathbb{R}^{2}$ is the set of points where the map is not proper (i.e., points that are limits of the map under sequences tending to infinity). In particular, a local diffeomorphism is a global diffeomorphism if and only if this set is empty. Gwoździewicz (2000) and Campbell (arXiv:math/9812032 in 1998, 2011) calculated the asymptotic variety of two Pinchuk maps in details. Our aim in this paper is to do a similar work, i.e., to describe a Pinchuk map, but now from a different point of view.

Let $U \subset \mathbb{R}^{2}$ be an open connected set. Let $F=(p, q): U \subset \mathbb{R}^{2} \rightarrow \mathbb{R}^{2}$ be a $C^{2}$ local diffeomorphism. Let $H_{F}(x, y)=\left(p(x, y)^{2}+q(x, y)^{2}\right) / 2$ and consider the Hamiltonian system

$$
\dot{x}=-\left(H_{F}\right)_{y}(x, y), \quad \dot{y}=\left(H_{F}\right)_{x}(x, y),
$$

where the dot denotes derivative with respect to the time $t$. The singular points of system (1) are characterized by the following result, that we shall prove below.

Correspondence to: Francisco Braun

E-mail: franciscobraun@dm.ufscar.br 
Lemma 1. The singular points of system (1) are the zeros of $F$, each of them is a center of system (1).

The following is a generalization of the characterization of global invertibility of polynomial maps given by Sabatini (1998). This version is due to Braun and Llibre (arXiv:1706.02643 in 2017).

Theorem 2. Let $z_{0} \in U$ such that $F\left(z_{0}\right)=(0,0)$. The center $z_{0}$ of system (1) is global if and only if (i) $F$ is globally injective and (ii) $F(U)=\mathbb{R}^{2}$ or $F(U)$ is an open disc centered at the origin.

In the special case that $F$ is a polynomial map and $U=\mathbb{R}^{2}$, it follows that $F\left(\mathbb{R}^{2}\right)=\mathbb{R}^{2}$ provided $F$ is injective (Białynicki-Birula and Rosenlicht 1962). Hence, in this case, $z_{0}$ is a global center of (1) if and only if $F$ is globally injective. An application of this result was given by Braun et al. (2016).

Since the phase portrait on the Poincaré sphere of a Hamiltonian polynomial vector field having a global center is simple, i.e., at the infinite either it does not have singular points, or the infinite singular points are formed by two degenerate hyperbolic sectors (for Hamiltonian vector fields, the infinity contains only isolated singular points), it is interesting to know how complex can be the phase portrait of a non-global center of a Hamiltonian system (1).

In this paper we provide the qualitative global phase portrait of the Hamiltonian system (1) when $F$ is given by the Pinchuk map considered by Campbell $(1998,2011)$, after a translation in the target in order to have only a point $z_{0}$ such that $F\left(z_{0}\right)=(0,0)$. More precisely, we prove the following result.

Theorem 3. Let $F=(p, q): \mathbb{R}^{2} \rightarrow \mathbb{R}^{2}$, where $(p, q+208): \mathbb{R}^{2} \rightarrow \mathbb{R}^{2}$ is the Pinchuk map considered by Campbell $(1998,2011)$ (see the definition below). Then the phase portrait of the Hamiltonian system (1) in the Poincare disc is topologically equivalent to the phase portrait given in Fig. 1.

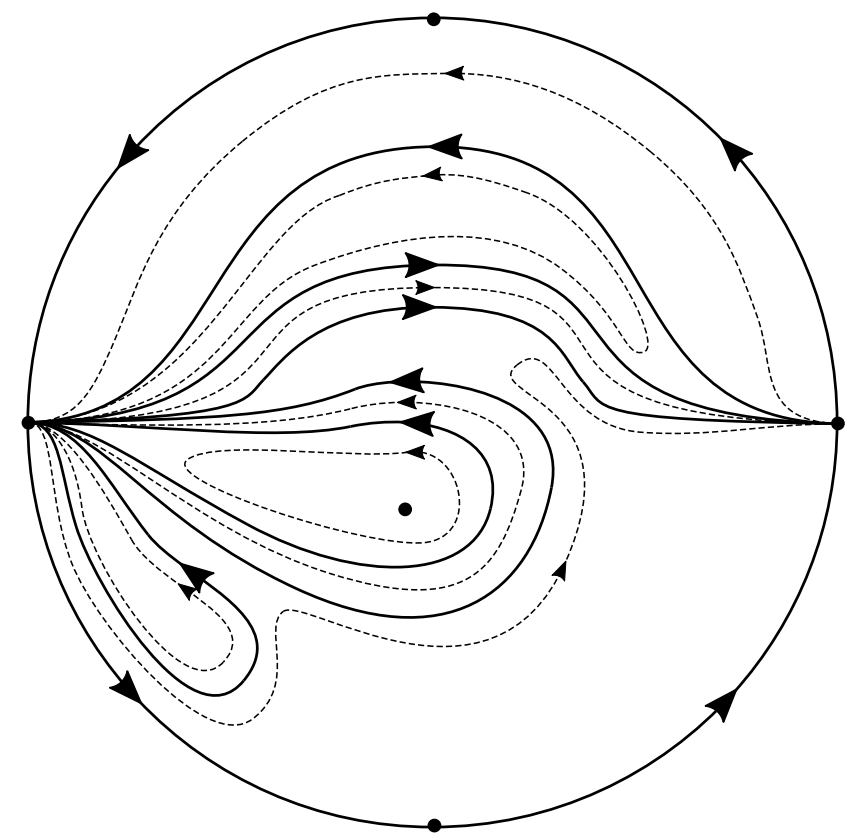

Figure 1 - The qualitative global phase portrait of system (1) in the Poincaré disc.

To prove Theorem 3, we first study the infinite singular points of system (1). These infinite singular points are very degenerate, and we apply homogeneous and quasi-homogeneous blow ups to study them. 
Then, we complete the proof of Theorem 3 by proving that the separatrix configuration of system (1) is qualitatively the one presented in Fig. 1.

We think that a good understanding of what fails in the real Jacobian conjecture could be interesting to investigate a related problem, the Jacobian conjecture in $\mathbb{R}^{2}$, that a polynomial local diffeomorphism whose Jacobian determinant is constant is globally injective. This conjecture remains unsolved until now. For the Jacobian conjecture we address the reader to the works of Bass et al. (1982) and Van Den Essen (2000).

\section{INJECTIVITY, CENTERS AND A PINCHUK MAP}

We begin with the proof of Lemma 1 .

Proof of Lemma 1. Let $z_{0}$ be a singular point of the Hamiltonian system (1). We have

$$
\left(\begin{array}{cc}
-p_{y}\left(z_{0}\right) & -q_{y}\left(z_{0}\right) \\
p_{x}\left(z_{0}\right) & q_{x}\left(z_{0}\right)
\end{array}\right)\left(\begin{array}{l}
p\left(z_{0}\right) \\
q\left(z_{0}\right)
\end{array}\right)=\left(\begin{array}{l}
0 \\
0
\end{array}\right),
$$

which is true if and only in $F\left(z_{0}\right)=(p, q)\left(z_{0}\right)=(0,0)$ because the Jacobian determinant of $F$ is nowhere zero.

The point $z_{0}$ is a center of the Hamiltonian system (1) because it is an isolated minimum of $H_{F}$.

Now, we select the map $F$ that we are going to work in this paper. Let $t=x y-1, h=t(x t+1)$ and $f=$ $(x t+1)^{2}\left(t^{2}+y\right)$. A Pinchuk map is a non-injective polynomial map with nowhere zero Jacobian determinant of the form $(P, Q): \mathbb{R}^{2} \rightarrow \mathbb{R}^{2}$ such that $P=h+f$ and $Q=-t^{2}-6 t h(h+1)-u(h, f)$, where $u$ is chosen so that $\operatorname{det} D(P, Q)(x, y)=t^{2}+(t+f(13+15 h))^{2}+f^{2}$. The following is the Pinchuk map studied by Campbell (1998, 2011):

$$
\begin{aligned}
& \bar{p}=h+f \\
& \bar{q}=-t^{2}-6 t h(h+1)-170 f h-91 h^{2}-195 f h^{2}-69 h^{3}-75 f h^{3}-\frac{75 h^{4}}{4} .
\end{aligned}
$$

According to Campbell (2011), the points $(-1,-163 / 4)$ and $(0,0) \in \mathbb{R}^{2}$ have no inverse image under $(\bar{p}, \bar{q})$, all the other points of the curve

$$
\gamma(s)=\left(s^{2}-1,-75 s^{5}+\frac{345 s^{4}}{4}-29 s^{3}+\frac{117 s^{2}}{2}-\frac{163}{4}\right), s \in \mathbb{R},
$$

which is a parametrization of the asymptotic variety of $(\bar{p}, \bar{q})$, have exactly one inverse image under this map, and the points of $\mathbb{R}^{2} \backslash \gamma(\mathbb{R})$ have two inverse images. Hence, in particular, the point $(0,208)$ has precisely one inverse image under $(\bar{p}, \bar{q})$.

We consider the map $F=(p, q): \mathbb{R}^{2} \rightarrow \mathbb{R}^{2}$ given by the translation

$$
p(x, y)=\bar{p}(x, y), \quad q(x, y)=\bar{q}(x, y)-208 .
$$


Observe that $F$ is a Pinchuk map according to our above-definition. Moreover, now there exists exactly one point $z_{0} \in \mathbb{R}^{2}$ such that $F\left(z_{0}\right)=(0,0)$. From Lemma 1 the point $z_{0}$ is the only finite singular point of system (1), corresponding to a non-global center of this system according to Theorem 2. Further, the curve

$$
\beta(s)=\gamma(s)-(0,208)=\left(s^{2}-1,-75 s^{5}+\frac{345 s^{4}}{4}-29 s^{3}+\frac{117 s^{2}}{2}-\frac{995}{4}\right),
$$

$s \in \mathbb{R}$, is the asymptotic variety of $F$, whose points have exactly one inverse image over $F$, but the points $(-1,-995 / 4)$ and $(0,208)$, which have none.

From now on, we restrict our attention to the specific Pinchuk map (2).

We first calculate the coordinates of the point $z_{0}$. Observe that $x t+1=x^{2} y-x+1$ is a factor of $p$. If this factor annihilates, then $h=0$ and $q=-t^{2}-208<0$. The other factor of $p$ is

$$
g(x, y)=-x+\left(1-2 x+3 x^{2}\right) y-x^{2}(-2+3 x) y^{2}+x^{4} y^{3}
$$

We observe that $g(0, y)=y$ and $q(0, y)=50 y-799 / 4$ do not annihilate at the same time, thus the first coordinate of the point $z_{0}$ is not 0 . Moreover, since the leading coefficient of $q(x, y)$ as a polynomial in $y$ is $-75 x^{15}$, it follows that the first coordinate of $z_{0}$ will be a point where the resultant in $y$ between $g(x, y)$ and $q(x, y)$ is zero. This resultant is the cubic $c(x)=31008391-11757152 x-155580672 x^{2}+2239078400 x^{3}$ multiplied by $-x^{36} / 64$. The discriminant of $c(x)$ is negative, so it has only one real root, which will be the first coordinate of the point $z_{0}$.

Repeating a similar reasoning now looking $g$ and $q$ as polynomials in $x$, we calculate their resultant and obtain that its zero is the only real root of the cubic $c(y)=1789023641600+100675956992 y+$ $26252413280 y^{2}+1506138481 y^{3}$, which will be the second coordinate of the point $z_{0}$.

Hence $z_{0}=(-0,22568337 \ldots,-17,491214 \ldots)$ approximately. Since $z_{0}$ is a center, the only finite singular point of system (1), near $z_{0}$ the phase portrait of this system is simple. Indeed, since $z_{0}$ is the minimum point of $H_{F}$, it follows that the gradient of $H_{F}$ points outward of each closed orbit of the center, and so each closed orbit of the center rotates in counterclockwise around $z_{0}$.

In the following section we shall investigate the infinite of system (1).

\section{THE INFINITE OF SYSTEM}

In this section, we will use results and notations on the Poincaré compactification of polynomial vector fields of $\mathbb{R}^{2}$. In particular $U_{i}, V_{i}, i=1,2,3$, are the canonical local charts of the Poincaré sphere $\mathbb{S}^{2}$.

For details on this technique we refer the reader to Chapter 5 of (Dumortier et al. 2006) or to (González Velasco 1969).

We call a singular point of a vector field linearly zero when the linear part of the vector field at this point is identically zero.

We begin by proving a general fact about the infinite singular points of Hamiltonian systems of the form (1). Writing $H=H_{0}+H_{1}+\cdots+H_{d+1}$, where $H_{i}$ is the homogeneous part of degree $i$ of the polynomial $H$, it is simple to conclude that the infinite singular points $(u, 0)$ of system (1) in the local charts $U_{1}$ and $U_{2}$ are 
the points satisfying $H_{d+1}(1, u)=0$ and $H_{d+1}(u, 1)=0$, respectively. Let $(u, 0)$ be an infinite singular point of system (1) and assume it is in the chart $U_{1}$. The linear part of the vector field at $(u, 0)$ is

$$
\left(\begin{array}{cc}
(d+1)\left(H_{d+1}\right)_{y}(1, u) & d H_{d}(1, u) \\
0 & \left(H_{d+1}\right)_{y}(1, u)
\end{array}\right) .
$$

Assuming $m=\operatorname{deg} p \geq \operatorname{deg} q$, we have $d=2 m-1$ and $H_{d}=p_{m} p_{m-1}+q_{m} q_{m-1}$ and $H_{d+1}=p_{m}^{2}+q_{m}^{2}$. Since $H_{d+1}(1, u)=0$, it follows that $p_{m}(1, u)=q_{m}(1, u)=0$, and hence $\left(H_{d+1}\right)_{y}(1, u)=H_{d}(1, u)=0$. Therefore, $(u, 0)$ is a linearly zero singular point. This proves the following result.

Lemma 4. The infinite singular points of the Hamiltonian system (1) are linearly zero.

Now, we return to the Pinchuk map $F$ defined by (2). Observe that the highest homogeneous part of $H_{F}(x, y)$ is $5625 x^{30} y^{20} / 2$. Thus, the origins of the charts $U_{1}, V_{1}$ and $U_{2}, V_{2}$ are the infinite singular points of the Hamiltonian system (1), each of them linearly zero from Lemma 4.

We will use the quasi-homogeneous directional blow up technique to desingularize each of these infinite singular points. An exposition about blow-ups can be found in (Álvarez et al. 2011), see also Chapter 3 of (Dumortier et al. 2006). We now recall the directional blow up transformations.

By the quasi-homogeneous blow up in the positive (resp. negative) $x$-direction with weights $\alpha$ and $\beta$, or simply $(\alpha, \beta)$-blow up in the positive (resp. negative) $x$-direction, we mean the transformation which carries the variables $\left(x_{1}, y_{1}\right)$ to the variables $\left(x_{2}, y_{2}\right)$ according to the formulas

$$
\left(x_{1}, y_{1}\right)=\left(x_{2}^{\alpha}, x_{2}^{\beta} y_{2}\right), \quad\left(x_{1}, y_{1}\right)=\left(-x_{2}^{\alpha}, x_{2}^{\beta} y_{2}\right),
$$

respectively. Similarly, by the quasi-homogeneous blow up in the positive (resp. negative) $y$-direction with weights $\alpha$ and $\beta$, or simply $(\alpha, \beta)$-blow up in the positive (resp. negative) $y$-direction, we mean the transformations

$$
\left(x_{1}, y_{1}\right)=\left(x_{2} y_{2}^{\alpha}, y_{2}^{\beta}\right), \quad\left(x_{1}, y_{1}\right)=\left(x_{2} y_{2}^{\alpha},-y_{2}^{\beta}\right),
$$

respectively.

Clearly if $\alpha$ (resp. $\beta$ ) is odd, then, the blow up in the positive $x$-direction (respec. $y$-direction) provides the information of the respectively negative blow ups. Also, if $\beta$ is odd, the $x$-directional blow ups swap the second and third quadrants, while the $y$-directional blow ups swap the third and the fourth quadrants if $\alpha$ is odd. After the $(\alpha, \beta)$-blow up in the $x$-direction, a system $\dot{x_{1}}=P\left(x_{1}, y_{1}\right), \dot{y_{1}}=Q\left(x_{1}, y_{1}\right)$ is transformed into

$$
\dot{x_{2}}=\frac{ \pm P}{\alpha x_{2}^{\alpha-1}}, \quad \dot{y_{2}}=\frac{\alpha x_{2}^{\alpha-1} Q \mp \beta x_{2}^{\beta-1} y_{2} P}{\alpha x_{2}^{\alpha+\beta-1}},
$$

with $P=P\left( \pm x_{2}^{\alpha}, x_{2}^{\beta} y_{2}\right)$ and $Q=Q\left( \pm x_{2}^{\alpha}, x_{2}^{\beta} y_{2}\right)$, in the positive and negative directions according to \pm . Similarly, the $(\alpha, \beta)$-blow up in the $y$-direction transforms $\dot{x_{1}}=P\left(x_{1}, y_{1}\right), \dot{y}_{1}=Q\left(x_{1}, y_{1}\right)$ into

$$
\dot{x_{2}}=\frac{\beta y_{2}^{\beta-1} P \mp \alpha x_{2} y_{2}^{\alpha-1} Q}{\beta y_{2}^{\alpha+\beta-1}}, \quad \dot{y_{2}}=\frac{ \pm Q}{\beta y_{2}^{\beta-1}},
$$


with $P=P\left(x_{2} y_{2}^{\alpha}, \pm y_{2}^{\beta}\right)$ and $Q=Q\left(x_{2} y_{2}^{\alpha}, \pm y_{2}^{\beta}\right)$, in the positive and in the negative directions according to \pm .

After the blow up in the $x$-direction (resp. $y$-direction) we cancel a common appearing factor $x_{2}^{k}\left(y_{2}^{k}\right)$ for a suitable $k$. So, if $k$ is odd, the direction of the orbits are reversed in $x_{2}<0\left(y_{2}<0\right)$.

The weights $\alpha$ and $\beta$ are chosen analyzing the Newton polygon of $(P, Q)$, see the construction in (Álvarez et al. 2011).

The application of $(\alpha, \beta)$-blow ups with $\alpha \beta \neq 1$ usually reduces the number of blow ups necessary for studying the local phase portrait of a linearly zero singular point.

To make the exposition clearer, we shall apply the most part of the blow ups in the $x$-direction. So, sometimes, we will first apply a xy-change, $\left(x_{1}, y_{1}\right) \mapsto\left(y_{1}, x_{1}\right)=\left(x_{2}, y_{2}\right)$, before making the blow-up.

In the next two subsections we will desingularize the origin of the charts $U_{1}$ and $U_{2}$, respectively. We will denote the coordinates of the system in the step $i$ of the algorithm as the variables $\left(w_{i}, z_{i}\right)$, so that after either a $w z$-change, a translation or a blow up, the new obtained system will be written in the variables $\left(w_{i+1}, z_{i+1}\right)$. In each step, we will denote the system $\dot{w}_{i}=P_{i}\left(w_{i}, z_{i}\right), \dot{z}_{i}=Q_{i}\left(w_{i}, z_{i}\right)$ simply as $\left(P_{i}, Q_{i}\right)$.

Since the Hamiltonian system (1) with the polynomials $p$ and $q$ given by (2) has degree 49, it follows that for the calculations in each step of the algorithm we have to deal with polynomials of very high degree. So, we persuade these calculations with the algebraic manipulator Mathematica. We do not show in each step the whole expressions of the systems $\left(P_{i}, Q_{i}\right)$ because this would be impractical.

\section{THE ORIGIN OF THE CHART $U_{1}$}

We write the compactification of system (1) in the chart $U_{1}$ in the variables $\left(w_{0}, z_{0}\right)$, as $\left(P_{0}, Q_{0}\right)$. From Lemma 4 , the singular point $(0,0)$ is linearly zero.

We first apply a $w z$-change and write the new system in the variables $\left(w_{1}, z_{1}\right)$ as $\left(P_{1}, Q_{1}\right)$.

The Newton polygon of system $\left(P_{1}, Q_{1}\right)$ has only one compact edge contained in the straight line $x+$ $2 y=38$. We apply $(1,2)$-blow ups in the positive $w$-direction and in the positive and negative $z$-directions obtaining systems $\left(P_{2}, Q_{2}\right)$ and $\left(P_{2}^{ \pm}, Q_{2}^{ \pm}\right)$, in the variables $\left(w_{2}, z_{2}\right)$ and $\left(w_{2}^{ \pm}, z_{2}^{ \pm}\right)$, after canceling the common factors $w_{2}^{38}$ and $\left(w_{2}^{ \pm}\right)^{38}$, respectively. The first terms of these systems have the following expressions:

$$
\begin{aligned}
P_{2} & =w_{2}\left(-56250+\frac{1125}{2}\left(447 w_{2}+1900 z_{2}\right)+\cdots\right) \\
Q_{2} & =28125-\frac{1125}{4}\left(387 w_{2}+2000 z_{2}\right)+\frac{75}{4}\left(1967 w_{2}^{2}+24138 w_{2} z_{2}+57000 z_{2}^{2}\right)+\cdots
\end{aligned}
$$

and

$$
\begin{aligned}
& P_{2}^{ \pm}=w_{2}^{ \pm}\left(\mp \frac{28125}{2}+281250\left(w_{2}^{ \pm}\right)^{2}+\cdots\right), \\
& Q_{2}^{ \pm}=z_{2}^{ \pm}\left( \pm \frac{140625}{2}-1350000\left(w_{2}^{ \pm}\right)^{2}+\cdots\right) .
\end{aligned}
$$

The only singular point of $\left(P_{2}, Q_{2}\right)$ over the line $w_{2}=0$ is the linearly zero singular point $(0,1)$. The origin of the systems $\left(P_{2}^{ \pm}, Q_{2}^{ \pm}\right)$are saddles as depicted in the planes $w_{2}^{+} z_{2}^{+}$and $w_{2}^{-} z_{2}^{-}$of Fig. 2 .

The reader can follow a schema of each step of the calculations in Fig. 2. We just need to analyze the origin of the systems $\left(P_{2}^{ \pm}, Q_{2}^{ \pm}\right)$, because the other singularities over the lines $z_{2}^{ \pm}=0$ will correspond to the singularity $(0,1)$ of $\left(P_{2}, Q_{2}\right)$. 


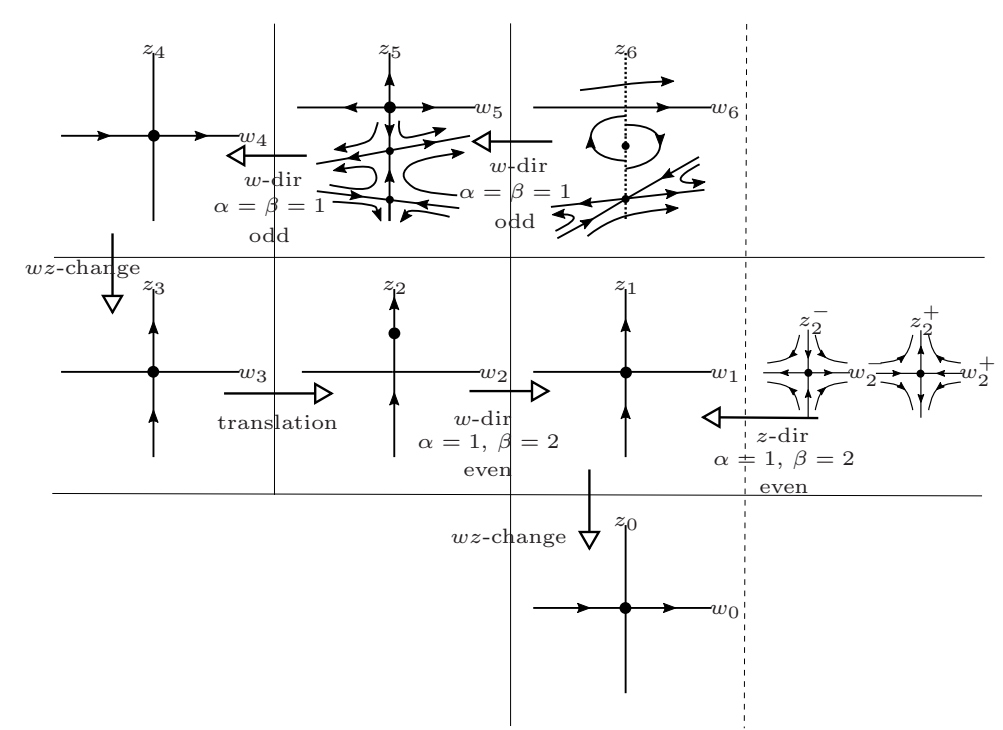

Figure 2 - The sequence of blow downs in the study of the origin of the chart $U_{1}$.

We now analyze this linearly zero singularity. We first do a translation bringing this point to the origin, obtaining the new system $\left(P_{3}, Q_{3}\right)$ in the variables $\left(w_{3}, z_{3}\right)$. We also apply a $w z$-change obtaining the system $\left(P_{4}, Q_{4}\right)$ in the variables $\left(w_{4}, z_{4}\right)$. The Newton polygon of this system has two compact edges. We choose the one contained in the straight line $x+y=11$. This compact edge has the point of negative abscissa $(-1,12)$, thus, concerning (1,1)-blow ups, it follows from Proposition 3.2 of (Álvarez et al. 2011) that $w_{4}$ is not a characteristic direction, and so we only need to apply a $w$-directional $(1,1)$-blow up, obtaining the system $\left(P_{5}, Q_{5}\right)$ in the variables $\left(w_{5}, z_{5}\right)$, after canceling the common factor $w_{5}^{11}$. The first terms of $\left(P_{5}, Q_{5}\right)$ are:

$$
\begin{aligned}
P_{5}= & w_{5}\left(\frac { 1 } { 4 } ( w _ { 5 } + z _ { 5 } ) ( w _ { 5 } + 2 z _ { 5 } ) \left(442125 w_{5}^{5} z_{5}+824250 w_{5}^{4} z_{5}^{2}+699990 w_{5}^{3} z_{5}^{3}\right.\right. \\
& \left.\left.+320532 w_{5}^{2} z_{5}^{4}+215904 w_{5} z_{5}^{5}+112500 w_{5}^{6}+217160 z_{5}^{6}\right)+\cdots\right), \\
Q_{5}= & z_{5}\left(\frac { 1 } { 4 } ( w _ { 5 } + z _ { 5 } ) ( w _ { 5 } + 2 z _ { 5 } ) \left(442125 w_{5}^{5} z_{5}+824250 w_{5}^{4} z_{5}^{2}+699990 w_{5}^{3} z_{5}^{3}\right.\right. \\
& \left.\left.+320532 w_{5}^{2} z_{5}^{4}+215904 w_{5} z_{5}^{5}+112500 w_{5}^{6}+217160 z_{5}^{6}\right)+\cdots\right) .
\end{aligned}
$$

Over the line $w_{5}=0$, the singular points of $\left(P_{5}, Q_{5}\right)$ are $(0,0)$ and two points of the form $\left(0, z_{5}\right)$, with $z_{5}$ the two real solutions of

$$
0=z_{5}^{4}+70726 z_{5}^{3}+252941 z_{5}^{2}+290380 z_{5}+108580
$$

The discriminant of this quartic equation is negative, thus, it has two real solutions. Those are approximately $z_{5}=-70722.424 \ldots$ and $z_{5}=-1.6611121 \ldots$ The singular point $(0,0)$ is linearly zero and the other two singular points are saddles, as represented in the $w_{5} z_{5}$-plane of Fig. 2.

Now, we study the linearly zero point $(0,0)$ of $\left(P_{5}, Q_{5}\right)$. It is clear from (4) that the characteristic equation of $\left(P_{5}, Q_{5}\right)$ is identically zero, so $(0,0)$ is a dicritical singular point. We apply $(1,1)$-blow ups in both the $w$ and $z$-directions obtaining systems $\left(P_{6}, Q_{6}\right)$ and $\left(P_{6}^{y}, Q_{6}^{y}\right)$ in the variables $\left(w_{6}, z_{6}\right)$ and $\left(w_{6}^{y}, z_{6}^{y}\right)$, after canceling 
the factors $w_{6}^{9}$ and $\left(z_{6}^{y}\right)^{9}$, respectively. System $\left(P_{6}^{y}, Q_{6}^{y}\right)$ does not have $(0,0)$ as a singular point, so we just need to consider system $\left(P_{6}, Q_{6}\right)$ over the line $w_{6}=0$. We have

$$
\begin{aligned}
P_{6}\left(0, z_{6}\right)= & \frac{1}{4}\left(z_{6}+1\right)\left(2 z_{6}+1\right)\left(217160 z_{6}^{6}+215904 z_{6}^{5}+320532 z_{6}^{4}+699990 z_{6}^{3}\right. \\
& \left.+824250 z_{6}^{2}+442125 z_{6}+112500\right), \\
Q_{6}\left(0, z_{6}\right)= & \frac{1}{4} z_{6}^{2}\left(z_{6}+1\right)\left(2 z_{6}+1\right)\left(290380 z_{6}^{6}+260416 z_{6}^{5}+421348 z_{6}^{4}+904140 z_{6}^{3}\right. \\
& \left.+1032225 z_{6}^{2}+542250 z_{6}+135000\right) .
\end{aligned}
$$

By using Sturm's theorem (see for instance (Isaacson and Keller 1994); in the software Mathematica, the Sturm theorem is programed by the instruction CountRoots) we see that the polynomial of degree 6 multiplying $\left(z_{6}+1\right)\left(2 z_{6}+1\right) / 4$ in $P_{6}\left(0, z_{6}\right)$ has no real roots, so, the only singular points are $(0,-1 / 2)$ and $(0,-1)$. The first one is a weak focus and the second one is a saddle, as depicted in the plane $w_{6} z_{6}$ of Fig. 2 . Since the origin of $\left(P_{5}, Q_{5}\right)$ is dicritical, it follows that each orbit crossing the line $w_{6}=0$ will correspond to two orbits tending to $(0,0)$ in positive or negative directions.

We now begin the process of blowing down.

It is simple to conclude that the phase portrait of the system $\left(P_{5}, Q_{5}\right)$ close to the origin is qualitatively the one depicted in (a) of Fig. 3. Consequently, by considering also the information close to the other two

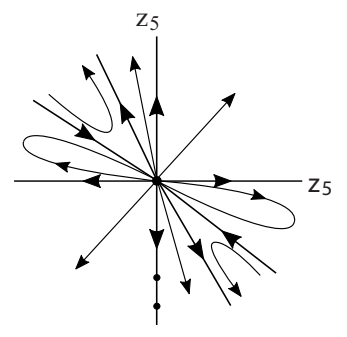

(a)

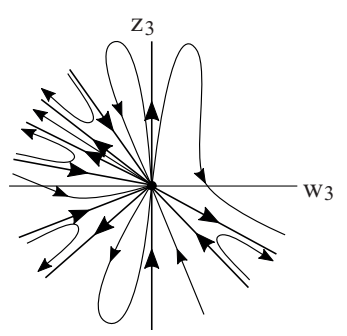

(b)

Figure 3 - The origin of system $\left(P_{5}, Q_{5}\right)$ in (a) and the origin of system $\left(P_{3}, Q_{3}\right)$ in (b).

singular points in the line $w_{5}=0$ (see the plane $w_{5} z_{5}$ of Fig. 2), we can understand the behavior near the origin of system $\left(P_{4}, Q_{4}\right)$. We then apply a wz-change and conclude that the behavior of system $\left(P_{3}, Q_{3}\right)$ near the origin is the one presented qualitatively in (b) of Fig. 3.

By translating $(0,0)$ to $(0,1)$ and by using the information provided by the saddles of planes $w_{2}^{ \pm} z_{2}^{ \pm}$, we make the blow downs with $\alpha=1$ and $\beta=2$, obtaining the origin of system $\left(P_{1}, Q_{1}\right)$. We then finally apply a $w z$-change and conclude that the origin of system $\left(P_{0}, Q_{0}\right)$ is qualitatively as drawn in Fig. 4.

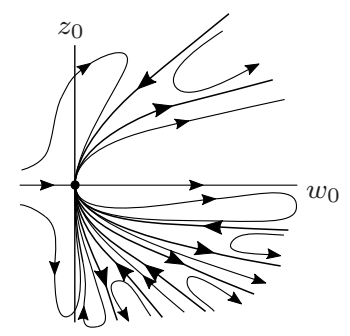

Figure 4 - The origin of the chart $U_{1}$. 


\section{THE ORIGIN OF CHART $U_{2}$}

As in the calculations made above, we write the compactified vector field in the chart $U_{2}$ as $\left(\dot{w_{0}}, \dot{z_{0}}\right)=$ $\left(P_{0}, Q_{0}\right)$. The Newton polygon of $\left(P_{0}, Q_{0}\right)$ has two compact edges: one of them contained in the straight line $3 x+2 y=87$. We apply a $(3,2)$-blow up in the $w$-direction, obtaining the system $\left(\dot{w_{1}}, \dot{z}_{1}\right)=\left(P_{1}, Q_{1}\right)$ after canceling the factor $w_{1}^{87}$. The first terms of $P_{1}$ and $Q_{1}$ are:

$$
\begin{aligned}
P_{1} & =w_{1}\left(-46875+11250 z_{1}^{2}\left(80 w_{1}-47 z_{1}\right)+\cdots\right) \\
Q_{1} & =z_{1}\left(9375+56250 z_{1}^{2}\left(2 z_{1}-3 w_{1}\right)+\cdots\right)
\end{aligned}
$$

The polynomials $P_{1}$ and $Q_{1}$ have degree 61 .

It is clear that at $(0,0)$ we have a saddle. The other singular point of $\left(P_{1}, Q_{1}\right)$ in the line $w_{1}=0$ is $(0,-1)$, and it is a linearly zero point. See the $w_{1} z_{1}$-plane of Fig. 5 . The reader can follow the steps of the calculations in the schema shown in this figure. We just warn that, differently of Fig. 2, we already draw the final phase portrait of each step, including the behavior close to the linearly zero points (information that we will know only after persuading all the blow ups).

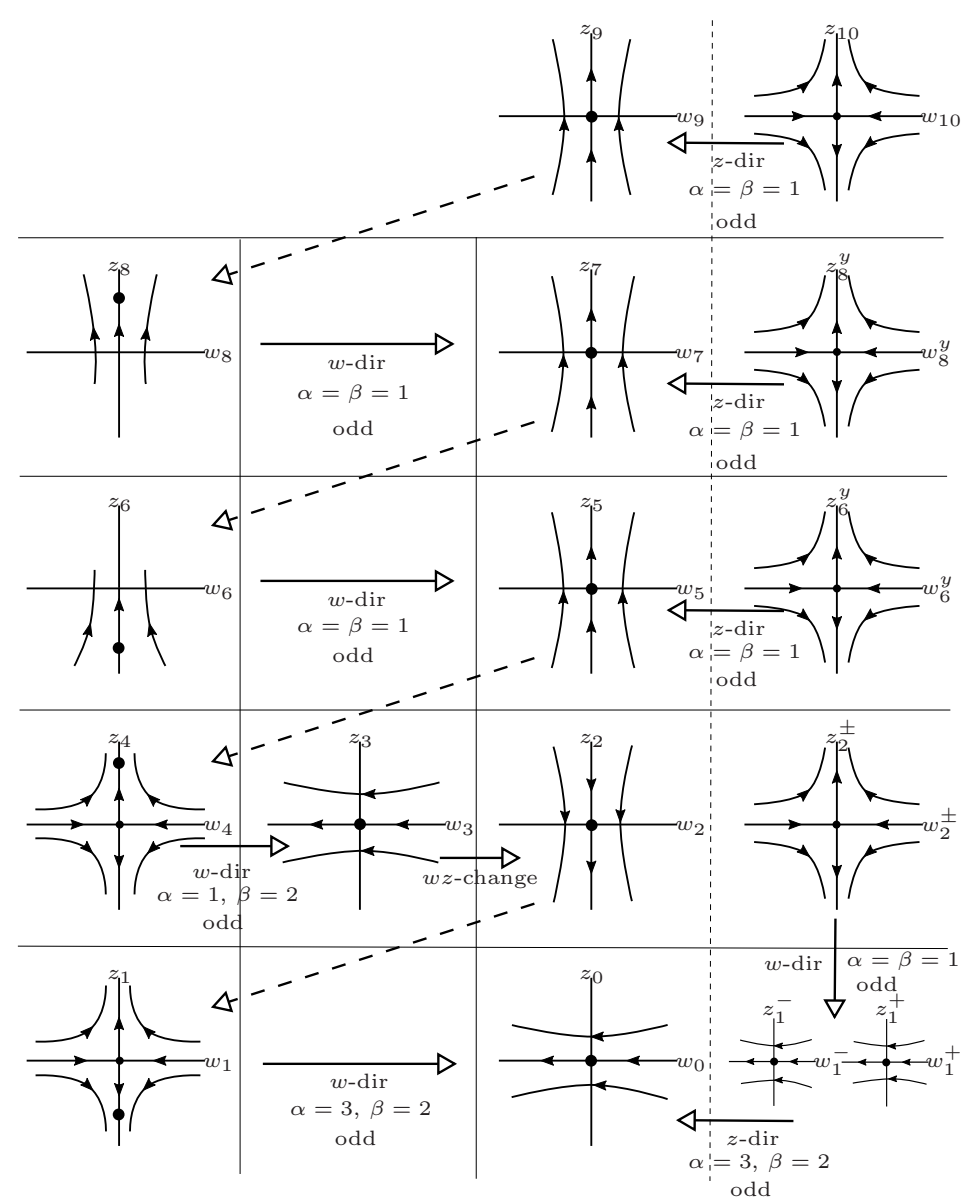

Figure 5 - The sequence of blow downs in the study of the origin of the chart $U_{2}$. 
We also apply $(3,2)$-blow ups in the positive and negative $z$-directions, obtaining the systems $w_{1}^{ \pm}=P_{1}^{ \pm}$, $z_{1}^{ \pm}=Q_{1}^{ \pm}$, respectively, with linearly zero singular points at $\left(w_{1}^{ \pm}, z_{1}^{ \pm}\right)=(0,0)$. The polynomials $P_{1}^{ \pm}$and $Q_{1}^{ \pm}$ have degree 30 and $Q_{1}^{ \pm}$is a factor of $z_{1}^{ \pm}$.

We do not need to analyze the other singular points over the lines $z_{1}^{ \pm}=0$, as the information provided by them is already contained in the $w$-directional blow up. We desingularize these points applying $(1,1)$-blow ups in the $w$-direction. Here, we do not need to apply blow ups in the $z$-directions because the characteristic equations of the systems are

$$
\begin{aligned}
0= & z_{1}^{ \pm}\left(-4500\left(w_{1}^{ \pm}\right)^{5} z_{1}^{ \pm}+1650\left(w_{1}^{ \pm}\right)^{4}\left(z_{1}^{ \pm}\right)^{2}-7800\left(w_{1}^{ \pm}\right)^{3}\left(z_{1}^{ \pm}\right)^{3}\right. \\
& \left.+3025\left(w_{1}^{ \pm}\right)^{2}\left(z_{1}^{ \pm}\right)^{4}-500 w_{1}^{ \pm}\left(z_{1}^{ \pm}\right)^{5}+5625\left(w_{1}^{ \pm}\right)^{6}+2501\left(z_{1}^{ \pm}\right)^{6}\right),
\end{aligned}
$$

and so $w_{1}^{ \pm}=0$ are not characteristic directions. We obtain the systems $\left(P_{2}^{ \pm}, Q_{2}^{ \pm}\right)$after canceling a factor $\left(w_{2}^{ \pm}\right)^{5}$. The polynomials $P_{2}^{ \pm}$and $Q_{2}^{ \pm}$have degree 45 , and up to order 2 they have the same expressions:

$$
\begin{aligned}
& P_{2}^{ \pm}=w_{2}^{ \pm}\left(-\frac{28125}{2}+10125 z_{2}^{ \pm}+\cdots\right), \\
& Q_{2}^{ \pm}=z_{2}^{ \pm}\left(5625-4500 z_{2}^{ \pm}+\cdots\right) .
\end{aligned}
$$

Thus, at $(0,0)$ the systems have a saddle, as depicted in the planes $w_{2}^{ \pm} z_{2}^{ \pm}$of Fig. 5. Moreover, any other singularity of the form $\left(0, z_{2}^{ \pm}\right)$must satisfy

$$
2501\left(z_{2}^{ \pm}\right)^{6}-500\left(z_{2}^{ \pm}\right)^{5}+3025\left(z_{2}^{ \pm}\right)^{4}-7800\left(z_{2}^{ \pm}\right)^{3}+1650\left(z_{2}^{ \pm}\right)^{2}-4500 z_{2}^{ \pm}+5625=0 .
$$

By using Sturm's theorem, we conclude that this equation has no real solution.

Now, we desingularize the point $(0,-1)$ of system $\left(P_{1}, Q_{1}\right)$. First, we apply a translation to bring this point to the origin, obtaining the system $\left(P_{2}, Q_{2}\right)$ in the variables $\left(w_{2}, z_{2}\right)$. We also apply a $w z$-change obtaining the system $\left(P_{3}, Q_{3}\right)$ in the variables $\left(w_{3}, z_{3}\right)$. The Newton polygon of this system has only one compact edge contained in the line $x+2 y=11$, and this edge has points of negative abscissa, so, concerning $(1,2)$ blow ups we just need to apply them in the $w$-direction, according to Proposition 3.2 of (Álvarez et al. 2011). Hence, we apply a $(1,2)$-blow up in the positive $w$-direction, obtaining the system $\left(P_{4}, Q_{4}\right)$ in the variables $\left(w_{4}, z_{4}\right)$, after canceling a factor of $w_{4}^{11}$. These polynomials have degree 90 , and their first terms are:

$$
\begin{aligned}
P_{4} & =w_{4}\left(-4982259375+\frac{996451875}{4}\left(260 w_{4}+z_{4}\right)+\cdots\right), \\
Q_{4} & =z_{4}\left(3985807500-110716875\left(639 w_{4}+2 z_{4}\right)+\cdots\right) .
\end{aligned}
$$

Clearly, $(0,0)$ is a singularity corresponding to a saddle. The other singular point in the line $w_{4}=0$ is $(0, \xi)$, where $\xi$ is the only real root of the cubic

$$
c(x)=4 x^{3}+216 x^{2}+6075 x-218700,
$$

which is approximately $\xi=18.8848 \ldots$. . This cubic has only one real root because its discriminant is negative. A calculation shows that $(0, \xi)$ is linearly zero. See the plane $w_{4} z_{4}$ in Fig. 5.

Now, we apply a translation to bring the point $(0, \xi)$ to the origin, obtaining system $\left(P_{5}, Q_{5}\right)$ written in the variables $\left(w_{5}, z_{5}\right)$. Since $\xi$ is not a rational number, we do this translation with a parameter $x$, and 
thus $P_{5}$ and $Q_{5}$ are polynomials in $w_{5}, z_{5}$ and $x$. We simplify these polynomials substituting them by the remainder of the division of each of them by $c(x)$, obtaining so polynomials of degree 2 in $x$, and hence when we substitute $x$ by $\xi$, we obtain the same expressions. We keep the notation $\left(P_{5}, Q_{5}\right)$.

The Newton polygon of this system has just one compact edge contained in the line $x+y=1$. So, the blow ups here will be homogeneous ones. The characteristic equation of system $\left(P_{5}, Q_{5}\right)$ is a multiple of

$$
\begin{aligned}
0= & w_{5}\left(729\left(23090824 x^{2}+532204875 x-18375684300\right) w_{5}^{2}\right. \\
& \left.-216\left(149 x^{2}+1828125 x-32221800\right) w_{5} z_{5}-4\left(404 x^{2}+8325 x-54675\right) z_{5}^{2}\right),
\end{aligned}
$$

with $x=\xi$. It would thus be enough to apply a $(1,1)$-blow up in the $z$-direction, and to study the singularities of the new system in $z_{6}=0$ (this could evidently also be concluded by observing that the compact edge of the Newton polygon of $\left(P_{5}, Q_{5}\right)$ has a point of negative ordinate). We prefer though to apply $(1,1)$-blow ups in the $w$ and $z$-directions and to study the singularities of the new systems either in the line $w_{6}=0$ and in the origin, respectively. The reason why we do this is that the singularities other than the origin are linearly zero and we have to apply new blow ups after persuading a translation. The matter here is that the blow up in the $z$-direction produces a vector field of degree 158, while the blow up in the $w$-direction produces a vector field of degree 109. Thus, it is simpler to do a translation and after to apply the polynomial remainder in the vector field with smaller degree.

Then, after applying $(1,1)$-blow ups in either the positive $w$ - and $z$-directions, we obtain the systems $\left(P_{6}, Q_{6}\right)$ and $\left(P_{6}^{y}, Q_{6}^{y}\right)$ in the variables $\left(w_{6}, z_{6}\right)$ and $\left(w_{6}^{y}, z_{6}^{y}\right)$, after canceling factors $w_{6}$ and $z_{6}^{y}$, respectively. The first terms of these systems are:

$$
\begin{aligned}
P_{6}= & w_{6}\left(\frac{59049}{4}\left(149 x^{2}+1828125 x-32221800\right)+\cdots\right), \\
Q_{6}= & -\frac{4782969}{16}\left(23090824 x^{2}+532204875 x-18375684300\right) \\
& +\frac{177147}{64}\left(5928191012 x^{2}-9644385686625 x+179165168144100\right) w_{6} \\
& +\frac{177147}{2}\left(149 x^{2}+1828125 x-32221800\right) z_{6}+\cdots,
\end{aligned}
$$

with $x=\xi$ and

$$
\begin{aligned}
& P_{6}^{y}=w_{6}^{y}\left(-\frac{6561}{4}\left(404 x^{2}+8325 x-54675\right)+\cdots\right), \\
& Q_{6}^{y}=z_{6}^{y}\left(2187\left(404 x^{2}+8325 x-54675\right)+\cdots\right),
\end{aligned}
$$

with $x=\xi$.

The origin of system $\left(P_{6}^{y}, Q_{6}^{y}\right)$ is a saddle (see the plane $w_{6}^{y} z_{6}^{y}$ in Fig. 5). On the other hand, the singularities of $\left(P_{6}, Q_{6}\right)$ over the line $w_{6}=0$ are the points $\left(0, z_{6}\right)$, with $z_{6}$ the real solutions of

$$
\begin{aligned}
0= & 4\left(404 x^{2}+8325 x-54675\right) z_{6}^{2}+216\left(149 x^{2}+1828125 x-32221800\right) z_{6} \\
& -729\left(23090824 x^{2}+532204875 x-18375684300\right),
\end{aligned}
$$

with $x=\xi$. The discriminant of this quadratic equation is a polynomial in $x$ whose division by $c(x)$ has remainder equal to 0 . This means that the only real solution of (5) is $r_{1}=-b /(2 a)$, where $a$ and $b$ are the 
coefficients of $z_{6}^{2}$ and $z_{6}$ in (5), respectively. Substituting $x$ by $\xi$ after applying the polynomial remainder again we have

$$
r_{1}=\frac{3\left(2380 \xi^{2}+21 \xi-334440\right)}{5989}
$$

The point $\left(0, r_{1}\right)$ is linearly zero, so, we translate it to the origin obtaining the system $\left(P_{7}, Q_{7}\right)$ in the variables $\left(w_{7}, z_{7}\right)$. We again persuade this translation considering $r_{1}=r_{1}(x)$ as a polynomial of $x$. Again $P_{7}$ and $Q_{7}$ will be polynomials in $w_{7}, z_{7}$ and $x$. As before we substitute these polynomials by the remainder of the division of them by $c(x)$, obtaining polynomials of degree 2 in $x$. We keep the notation $P_{7}$ and $Q_{7}$ for them.

The Newton polygon of this system has only one compact edge contained in the straight line $x+y=1$. The characteristic equation of this system has $w_{7}=0$ as a solution.

As above, we apply $(1,1)$-blow ups in either the positive $w$ - and $z$-directions, obtaining the systems $\left(P_{8}, Q_{8}\right)$ and $\left(P_{8}^{y}, Q_{8}^{y}\right)$ in the variables $\left(w_{8}, z_{8}\right)$ and $\left(w_{8}^{y}, z_{8}^{y}\right)$, respectively. We then study the origin of $\left(P_{8}^{y}, Q_{8}^{y}\right)$ and the singularities of $\left(P_{8}, Q_{8}\right)$ over the line $w_{8}=0$. The reason is again computational, as the degree of $\left(P_{8}^{y}, Q_{8}^{y}\right)$ is 196 and the degree of $\left(P_{8}, Q_{8}\right)$ is 128 . The first terms of these systems are:

$$
\begin{aligned}
P_{8}= & w_{8}\left(-\frac{6561\left(610023097091 x^{2}-7154910819000 x-72219849901200\right)}{95824}+\cdots\right), \\
Q_{8}= & \frac{59049}{4591119488}\left(866106385697199684752 x^{2}-63678825997496319079125 x\right. \\
& +894244583851567110026100)+\cdots,
\end{aligned}
$$

with $x=\xi$, and

$$
\begin{aligned}
& P_{8}^{y}=w_{8}\left(-\frac{2187}{2}\left(404 x^{2}+8325 x-54675\right)+\cdots\right), \\
& Q_{8}^{y}=z_{8}\left(\frac{6561}{4}\left(404 x^{2}+8325 x-54675\right)+\cdots\right),
\end{aligned}
$$

with $x=\xi$.

System $\left(P_{8}^{y}, Q_{8}^{y}\right)$ has a saddle at the origin (see the plane $w_{8}^{y} z_{8}^{y}$ in Fig. 5), while the singular points of $\left(P_{8}, Q_{8}\right)$ over the line $w_{8}=0$ are the points $\left(0, z_{8}\right)$, with $z_{8}$ the real roots of

$$
\begin{aligned}
0= & 2295559744\left(404 x^{2}+8325 x-54675\right) z_{8}^{2}-574944\left(610023097091 x^{2}\right. \\
& -7154910819000 x-72219849901200) z_{8}+27\left(866106385697199684752 x^{2}\right. \\
& -63678825997496319079125 x+894244583851567110026100),
\end{aligned}
$$

with $x=\xi$. The discriminant of this equation is a polynomial in $x$ whose division by $c(x)$ has remainder 0 . Thus, the only real solution is $r_{2}=-b /(2 a)$, where $a$ and $b$ are the coefficients of $z_{8}^{2}$ and $z_{8}$ of the equation, respectively. After applying the polynomial remainder, we substitute $x$ by $\xi$ obtaining

$$
r_{2}=\frac{-38570325688 \xi^{2}-1361034154573 \xi+41691943772820}{430417452} .
$$

The point $\left(0, r_{2}\right)$ is linearly zero, so, we translate it to the origin obtaining the system $\left(P_{9}, Q_{9}\right)$ in the variables $\left(w_{9}, z_{9}\right)$. As before, we make this translation with the parameter $x$, so that $P_{9}$ and $Q_{9}$ are polynomials in $x$. Keeping the notation we substitute these polynomials by the remainder of the division of them by $c(x)$. 
As before the Newton polygon of this system has only one compact edge contained in the straight line $x+y=1$. Moreover, the characteristic equation does not have $z_{9}=0$ as a solution. Here, we just apply a $(1,1)$-blow up in the positive $z$-direction, obtaining system $\left(P_{10}, Q_{10}\right)$ in the variables $\left(w_{10}, z_{10}\right)$, after canceling the factor $z_{10}$ (here we do not use the superscript $y$ as this is the only system in this step). The degree of this new system is 234 , but as we are going to see, just the origin is a singular point in the line $z_{10}=0$. The first terms of $P_{10}$ and $Q_{10}$ are:

$$
\begin{aligned}
P_{10} & =w_{10}\left(-\frac{2187}{4}\left(404 x^{2}+8325 x-54675\right)+\cdots\right), \\
Q_{10} & =z_{10}\left(\frac{2187}{2}\left(404 x^{2}+8325 x-54675\right)+\cdots\right),
\end{aligned}
$$

with $x=\xi$.

Now, over the line $z_{10}=0$, the singular points of $\left(P_{10}, Q_{10}\right)$ are $(0,0)$ and the points $\left(w_{10}, 0\right)$, with $w_{10}$ the real roots of

$$
\begin{aligned}
0= & 27\left(224799605593831132981000196646508 x^{2}\right. \\
& +11060763183198622719418769173796625 x \\
& -289048399074933337876985160926408100) w_{10}^{2} \\
& -1721669808\left(375535867201456283 x^{2}+10785776535503894250 x\right. \\
& -338993606077717260600) w_{10}+41168707330260512\left(404 x^{2}+8325 x-54675\right),
\end{aligned}
$$

with $x=\xi$. The discriminant of this equation after applying the polynomial remainder is

$$
\begin{aligned}
\Delta(x)= & 42795139080321190650757595864867731660278486158784 x^{2} \\
& +2546081344010178238089386481604981090589087283168000 x \\
& -63345629158853164845226783632142224359340633182668800 .
\end{aligned}
$$

It is simple to conclude that $\Delta(\xi)<0$, thus, only $(0,0)$ is a singular point of $\left(P_{10}, Q_{10}\right)$ in $z_{10}=0$. This singular point is the saddle depicted in the plane $w_{10} z_{10}$ of Fig. 5.

Since the behavior near each appearing singular points in each step above is very simple, the blow down of each step is also very simple: following the arrays in Fig. 5, it is easy to conclude that the origin of $U_{2}$ has a degenerate hyperbolic sector as shown in the $w_{0} z_{0}$-plane of Fig. 5.

\section{THE GLOBAL PHASE PORTRAIT}

We begin with a background on separatrices and canonical regions of the Poincare compactification $p(\mathscr{X})$ in the Poincaré disc $\mathbb{D}$ of a polynomial system $\dot{x}=\mathscr{X}(x)$. Let $\varphi$ be the flow of $p(\mathscr{X})$ defined in $\mathbb{D}$. As usual we denote by $(U, \varphi)$ the flow of $p(\mathscr{X})$ on an invariant subset $U \subset \mathbb{D}$. Two flows $(U, \varphi)$ and $(V, \psi)$ are said to be topologically equivalent if there exists a homeomorphism $h: U \rightarrow V$ sending orbits of $(U, \varphi)$ onto orbits of $(V, \psi)$ preserving or reversing the orientation of all the orbits.

Following Markus (1954), we say that the flow $(U, \varphi)$ is parallel if it is topologically equivalent to one of the following flows: (i) the flow defined in $\mathbb{R}^{2}$ by the system $\dot{x}=1, \dot{y}=0$; (ii) the flow defined in $\mathbb{R}^{2} \backslash\{(0,0)\}$ by the system in polar coordinates $\dot{r}=0, \dot{\theta}=1$; and (iii) the flow defined in $\mathbb{R}^{2} \backslash\{(0,0)\}$ by 
the system in polar coordinates $\dot{r}=r, \dot{\theta}=0$. Parallel flows topologically equivalent to (i), (ii) and (iii) are called strip, annular and spiral (or radial), respectively.

We denote by $\gamma_{x}$ the orbit of $p(\mathscr{X})$ passing through $x$ when $t=0$ with maximal interval $I_{x}$, and the positive (resp. negative) orbit of $\gamma_{x}$ by $\gamma_{x}^{+}=\left\{\gamma_{x}(t) \mid t \in I_{x}\right.$ and $\left.t \geq 0\right\}$ (resp. $\gamma_{x}^{-}=\left\{\gamma_{x}(t) \mid t \in I_{x}\right.$ and $\left.t \leq 0\right\}$ ). Then we set $a^{ \pm}(x)=\overline{\gamma_{x}^{ \pm}} \backslash \gamma_{x}^{ \pm}$, here as usual $\overline{\gamma_{x}^{ \pm}}$denotes the closure of $\gamma_{x}^{ \pm}$. Observe that $a^{-}(x)$ differs from $\alpha(x)$ in the case of periodic orbits and singular points: indeed, $a^{-}(x)=\emptyset$ and $\alpha(x)=\gamma_{x}$ in this case (similarly for $a^{+}(x)$ and $\left.\omega(x)\right)$. An orbit $\gamma_{x}$ of $p(\mathscr{X})$ is called a separatrix of $p(\mathscr{X})$ if it is not contained in an open neighborhood $U$ such that $(U, \varphi)$ is parallel and such that both $a^{ \pm}(x)=a^{ \pm}(y)$ for all $y \in U$ and $\bar{U} \backslash U$ consists of $a^{+}(x), a^{-}(x)$ and exactly two orbits $\gamma_{y}$ and $\gamma_{z}$ such that $a^{ \pm}(x)=a^{ \pm}(y)=a^{ \pm}(z)$.

If $\mathscr{X}$ is a polynomial vector field it is known that the separatrices of $p(\mathscr{X})$ are (i) the finite and infinite singular points of $p(\mathscr{X})$; (ii) the orbits of $p(\mathscr{X})$ contained in the boundary $\mathbb{S}^{1}$ of $\mathbb{D}$; (iii) the limit cycles of $p(\mathscr{X})$; and (iv) the separatrices of the hyperbolic sectors of the finite and infinite singular points of $p(\mathscr{X})$. Moreover, if $p(\mathscr{X})$ has finitely many finite and infinite singular points and finitely many limit cycles, then $p(\mathscr{X})$ has finitely many separatrices. We call each connected component of the complement of the union of separatrices a canonical region of $p(\mathscr{X})$. Neumann (1975) proved that each canonical region of a vector field $p(\mathscr{X})$ is parallel.

To the union of the separatrices of $p(\mathscr{X})$ together with an orbit belonging to each canonical region of $p(\mathscr{X})$ we call a separatrix configuration of $p(\mathscr{X})$. We say that the separatrix configurations $S_{1}$ and $S_{2}$ of $p\left(\mathscr{X}_{1}\right)$ and $p\left(\mathscr{X}_{2}\right)$ are topologically equivalent if there exists an orientation preserving homeomorphism from $\mathbb{D}$ to $\mathbb{D}$ which transforms orbits of $S_{1}$ onto orbits of $S_{2}$. The following is the Markus-Neumann-Peixoto classification theorem (Markus 1954, Neumann 1975, Neumann and O’Brien 1976, Peixoto 1973, Dumortier et al. 2006) for the Poincaré compactification in the Poincaré disc of polynomial systems.

Theorem 5 (Markus-Neumann-Peixoto). Let $p\left(\mathscr{X}_{1}\right)$ and $p\left(\mathscr{X}_{2}\right)$ be the Poincaré compactification of two polynomial systems $\dot{x}=\mathscr{X}_{1}(x)$ and $\dot{x}=\mathscr{X}_{2}(x)$, respectively. The flows of $p\left(\mathscr{X}_{1}\right)$ and $p\left(\mathscr{X}_{2}\right)$ on the Poincaré disc are topological equivalent if and only if the separatrix configurations of $p\left(\mathscr{X}_{1}\right)$ and $p\left(\mathscr{X}_{2}\right)$ are topological equivalent.

Hence, in order to qualitatively describe the phase portrait on the Poincare disc of system (1) it is enough to qualitatively describe its separatrix configuration. This was done in Fig. 1, where we have drawn the separatrices other than singular points with bold lines. The other lines are orbits contained in its respective canonical regions. We observe from Fig. 1 that system (1) has 15 separatrices, five of them singular points, and 7 canonical regions, six of them of type strip and the one formed by the closed orbits surrounding $z_{0}$, annular.

Below, we prove Theorem 3 by proving that Fig. 1 is a separatrix configuration of system (1).

From the previous sections we conclude that close enough to the singular points, the phase portrait of system (1) is qualitatively the one presented in Fig. 6. For further references we label the hyperbolic, parabolic and elliptic sectors presenting in the origins of the charts $U_{1}$ and $V_{1}$ in Fig. 6 as $h_{1}, h_{2}, h_{3}, h_{4}, p_{1}$, $p_{2}$ and $e_{1}, e_{2}$, respectively.

From the definition of system (1), each of its orbits is a connected component of a level set of $H_{F}=$ $\left(p^{2}+q^{2}\right) / 2$ (because the only singular point of this system is the center $z_{0}$ ), which in turn is the inverse image under $F=(p, q)$ of circles surrounding the point $(0,0)$. Since $F$ preserves orientation (because the Jacobian determinant of $F$ is positive), each orbit of (1) is carried onto a curve contained in a circle with 


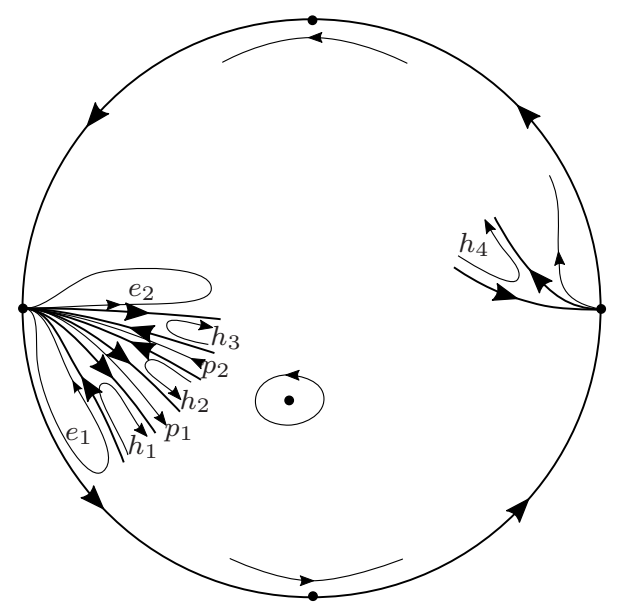

Figure 6 - The phase portrait of system (1) near the singular points.

counterclockwise orientation. As we have seen, the curve $\beta(s)$ defined in (3) is the asymptotic variety of $F$. Moreover, the points $\beta(0)=(-1,-995 / 4)$ and $\beta(1)=(0,208)$ of this curve have no inverse image under $F$, all the other points of this curve have exactly one inverse image and the other points of $\mathbb{R}^{2}$ have precisely two inverse images. Acting as Campbell (arXiv:math/9812032 1998), we delete from the curve $\beta(s)$ the points $\beta(0)$ and $\beta(1)$, obtaining three curves: $C_{1}=\beta(-\infty, 0), C_{2}=\beta(0,1)$ and $C_{3}=\beta(1, \infty)$. According to Campbell (1998), the inverse image under $F$ of each $C_{i}$ is a curve that divides the plane into two connected components. We call $D_{i}$ the inverse image of $C_{i}, i=1,2,3$. The set $D_{1} \cup D_{2} \cup D_{3}$ is called by Campbell (1998) the asymptotic flower of $F$. It follows that $\mathbb{R}^{2} \backslash\left(D_{1} \cup D_{2} \cup D_{3}\right)$ is formed by 4 connected components, each of them mapped twice onto each of the two connected components of $\mathbb{R}^{2} \backslash\{\beta(s)\}$. Each curve $C_{i}$ has a natural orientation, given by its parametrization (it is the opposite orientation used by Campbell (1998)). So, each curve $D_{i}$ also has a natural orientation (recall that $F$ preserves orientation). The graphics of $C_{i}$ and $D_{i}, i=1,2,3$, are given in (a) and (b) of Fig. 7, respectively. As in (Campbell 1998, 2011) the axes in (a) have different scales. Following Campbell (1998), we label the regions as $R$ (right) and $L$ (left) of the curves $C_{i}$ and $D_{i}$.

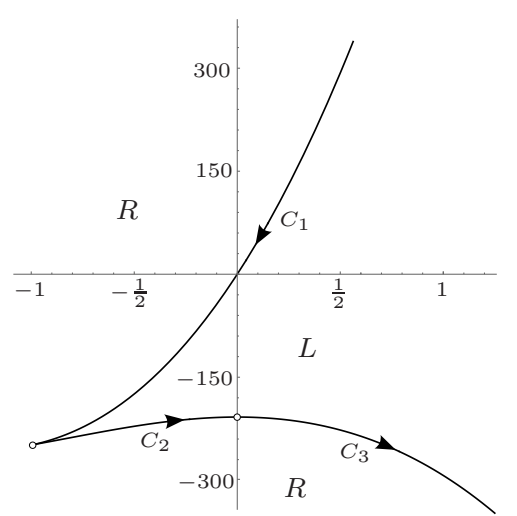

(a)

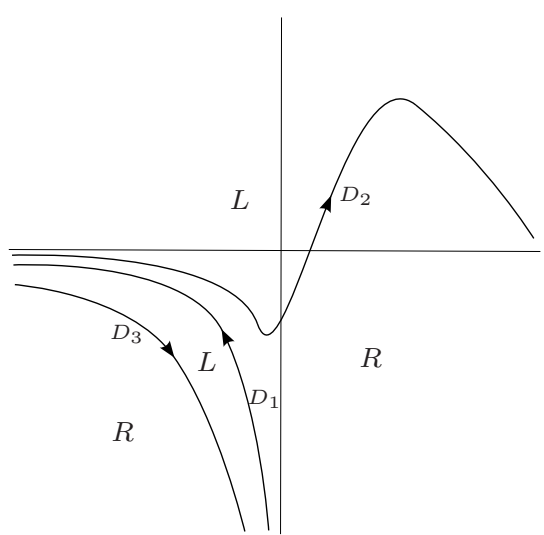

(b)

Figure 7 - The asymptotic variety of $F$ in (a) and the asymptotic flower of $F$ in (b). 
Since for each $s \in \mathbb{R}$

$$
\begin{aligned}
\beta^{\prime}(s) \cdot \beta(s)= & \frac{1}{4}(1-s) s(s+1)\left(112500 s^{6}-232875 s^{5}+301125 s^{4}-425760 s^{3}\right. \\
& \left.+432312 s^{2}-86565 s+116423\right)
\end{aligned}
$$

and this polynomial of degree 6 multiplying $(1-s) s(s+1)$ has no real zeros by Sturm's theorem, it follows that the curves $C_{1}, C_{2}$ and $C_{3}$ are transversal to the circles centered at $(0,0)=\beta(-1)$. As a consequence the curves $D_{1}, D_{2}$ and $D_{3}$ are transversal to the non-singular orbits of system (1). In particular, the image of a non-periodic orbit of system (1) has $\alpha$-and $\omega$-limits contained in the curve $\beta(s)$. Below, we will say that the image of an orbit starts or finishes at $\beta\left(s_{0}\right)$ meaning that its $\alpha$ - or $\omega$-limit is $\beta\left(s_{0}\right)$, respectively. Moreover, through each point in the intersection of $C_{1} \cup C_{2} \cup C_{3}$ with a circle, it crosses exactly one image of an orbit of system (1).

We call $S_{1}$ and $S_{2}$ the circles centered at $(0,0)$ and containing the points $\beta(1)$ and $\beta(0)$, respectively.

The point $z_{0}$, being the inverse image under $F$ of $(0,0)=\beta(-1)$, is contained in the curve $D_{1}$. The images under $F$ of the closed orbits surrounding $z_{0}$ are circles surrounding $(0,0)$ contained in the bounded region defined by $S_{1}$. Thus the boundary of the period annulus of the center $z_{0}$ corresponds to the arc of circle contained in $S_{1}$, starting and finishing at the point $\beta(1)$. This means that the boundary of the period annulus is an orbit that goes to infinity through the region labeled by $L$ in (b) of Fig. 7. In particular, in the Poincare disc, this orbit tends to the origin of the chart $V_{1}$. Then, analyzing the possibilities in Fig. 6 , we see that this orbit contains the two separatrices of the hyperbolic sector $h_{2}$. This period annulus is an annular canonical region.

Now we analyze the parabolic sectors $p_{1}$ and $p_{2}$.

Close to the two points of $D_{1}$ cut by the orbit giving the boundary of the period annulus of the center (i.e., the orbit connecting the two separatrices of the hyperbolic sector $h_{2}$ ), and outside the period annulus, there must exist orbits cutting $D_{1}$. Analyzing the images of these orbits, they are contained in circles surrounding the circle $S_{1}$. So, there are two possibilities for the images of these orbits: either they are arcs starting and finishing at a point of the curve $C_{2}$, or they are arcs starting at the curve $C_{3}$ and finishing at the curve $C_{2}$ or $C_{3}$. At a first glance both of these possibilities are compatible with the parabolic sectors $p_{1}$ and $p_{2}$ in Fig. 6. We claim that the correct possibility is the first one. Indeed, we can increase the radii of these circles containing the images of the orbits of $p_{1}$ and $p_{2}$ until we achieve the circle $S_{2}$. If we are in the second possibility, the orbit whose image is contained in $S_{2}$ and starts at a point of $C_{3}$ will contain the separatrice of the end of the parabolic sector $p_{2}$. But, this orbit will not contain the separatrice of the end of the parabolic sector $p_{1}$, because we can continue drawing arcs starting at $C_{3}$ with radii bigger than the radius of $S_{2}$. Thus, the parabolic sector $p_{1}$ will not finish, a contradiction with the nature of the vector field at the origin of the chart $V_{1}$, as shown in Fig. 6. This proves the claim.

So, the image of the orbits of the parabolic sectors $p_{1}$ and $p_{2}$ are arcs starting and finishing at a point of the curve $C_{2}$. And since we can continue drawing these arcs until we arrive at circle $S_{2}$, this means that the parabolic sectors $p_{1}$ and $p_{2}$ are connected, and the image of the orbit containing the separatrices that separate $p_{1}$ from $h_{1}$ and $p_{2}$ from $h_{3}$ is contained in the arc of $S_{2}$ starting and finishing at the point $\beta(0)$. The region connecting $p_{1}$ to $p_{2}$ is a strip canonical region, see Fig. 1.

Now, since the image of this last orbit cuts the curves $C_{3}$ and $C_{1}$, there must exist orbits near it whose images cross $C_{3}$ and $C_{1}$. The only possibility is that those images are arcs of circles starting at the curve $C_{1}$, 
rotating a complete turn crossing $C_{3}$ and $C_{1}$ and continue up to finishing in the curve $C_{3}$. We call these orbits the big orbits. A big orbit whose image is contained in a circle close enough to $S_{2}$ enters both the hyperbolic sectors $h_{1}$ and $h_{3}$. We have to see where the big orbits start and finish.

The orbits whose images are arcs of the circles with radii smaller than the radius of $S_{2}$, starting and finishing at $C_{1}$ and contained in the region $R$ correspond to an elliptic sector with boundary formed by an orbit having image contained in the arc of $S_{2}$ starting at $C_{1}$ an finishing at $\beta(0)$. Close to this boundary and out of the elliptic sector there must exist orbits whose images start at $C_{1}$. These orbits are the big orbits. Hence, it follows that this elliptic sector is $e_{1}$ and that the big orbits start at the origin of $V_{1}$, see Fig. 1 .

The orbits whose images are arcs starting and finishing at $C_{1}$ and contained in the region $L$ form the elliptic sector $e_{2}$. Clearly its boundary is formed by the two orbits containing the separatrices of the hyperbolic sector $h_{4}$. The image of these orbits are the arcs starting at $C_{1}$ and finishing at $\beta(1)$ and starting at $\beta(1)$ and finishing at $C_{1}$, respectively.

In particular, this means that the big orbits must finish at the origin of the chart $U_{1}$, below the hyperbolic sector $h_{4}$. Since their images are contained in the circles bigger than $S_{2}$, there exist orbits whose images are arcs contained in the circles between $S_{1}$ and $S_{2}$, starting at $C_{1}$, crossing $C_{2}$ and finishing at $C_{3}$. These orbits produce a parabolic sector between $h_{3}$ and $e_{2}$, and give rise to a strip canonical region as presented in Fig. 1 .

The big orbits also produce a strip canonical region.

The orbits of the strip canonical region placed above the hyperbolic sector $h_{4}$ have their images contained in arcs of circles with radii bigger than the radius of $S_{1}$, starting at $C_{3}$ and finishing at $C_{1}$.

The elliptic sectors $e_{1}$ and $e_{2}$ form another two strip canonical regions.

Hence we have 7 canonical regions, six of them are strip and one is annular. Analyzing Fig. 1, we see there are 6 finite orbits that are separatrices. The infinite has another 4 orbits. Hence, since there are 5 singular points, we have 15 separatrices in the separatrix configuration of system (1) in the Poincaré disc.

\section{ACKNOWLEDGMENTS}

The first and third authors are partially supported by the Ministerio de Economia y Competitividad (MINECO) grant number MTM2013-40998-P, the Agència de Gestió d'Ajuts Universitaris i de Recerca (AGAUR) grant number 2014SGR 568 and the grants from European Commission (FP7-PEOPLE-2012IRSES) numbers 316338 and 318999. The second author is partially supported by Fundação de Amparo à Pesquisa do Estado de São Paulo (FAPESP), grant number 2014/ 26149-3. The second and third authors are also partially supported by Coordenação de Aperfeiçoamento de Pessoal de Nível Superior (CAPES), grant 88881. 030454/ 2013-01 from the program CSF-PVE.

\section{REFERENCES}

ÁLVAREZ MJ, FERRAGUT A AND JARQUE X. 2011. A survey on the blow up technique. Internat J Bifur Chaos Appl Sci Engrg 21: 3103-3118.

BASS H, CONNEL EH AND WRIGHT D. 1982. The Jacobian conjecture: reduction of degree and formal expansion of the inverse. Bull Amer Math Soc 7: 287-330.

BIAŁYNICKI-BIRULA A AND ROSENLICHT M. 1962. Injective morphisms of real algebraic varieties. Proc Amer Math Soc 13: 200-203.

BRAUN F, GINÉ J AND LLIBRE J. 2016. A sufficient condition in order that the real Jacobian conjecture in $\mathbb{R}^{2}$ holds. J Differential Equations 260: 5250-5258.

CAMPBELL LA. 2011. The asymptotic variety of a Pinchuk map as a polynomial curve. Appl Math Lett 24: 62-65. 
DUMORTIER F, LLIBRE J AND ARTÉS JC. 2006. Qualitative theory of planar differential systems. Universitext, Springer-Verlag, $298 \mathrm{p}$.

GONZÁLEZ VELASCO EA. 1969. Generic properties of polynomial vector fields at infinity. Trans Amer Math Soc 143: $201-222$. GWOŹDZIEWICZ J. 2000. A geometry of Pinchuk's map. Bull Polish Acad Sci Math 48: 69-75.

ISAACSON E AND KELLER HB. 1994. Analysis of numerical methods, Corrected reprint of the 1966 original Wiley. New York: Dover Publications, $541 \mathrm{p}$.

MARKUS L. 1954. Global structure of ordinary differential equations in the plane. Trans Amer Math Soc 76: 127-148.

NEUMANN DA. 1975. Classification of continuous flows on 2-manifolds. Proc Amer Math Soc 48: 73-81.

NEUMANN DA AND O'BRIEN T. 1976. Global structure of continuous flows on 2-manifolds. J Differ Equations 22: 89-110.

PEIXOTO MM. 1973. On the classification of flows on 2-manifolds. Dynamical systems (Proc. Sympos., Univ. Bahia, Salvador, 1971). New York: Academic Press, p. 389-419.

PINCHUK S. 1994. A counterexample to the strong real Jacobian conjecture. Math Z 217: 1-4.

SABATINI M. 1998. A connection between isochronous Hamiltonian centres and the Jacobian conjecture. Nonlinear Anal 34: 829-838.

VAN DEN ESSEN A. 2000. Polynomial automorphisms and the Jacobian conjecture. Progress in Mathematics, 190. Basel: Birkhäuser Verlag, 329 p. 